Published in final edited form as:

Leukemia. 2020 March ; 34(3): 872-881. doi:10.1038/s41375-019-0623-5.

\title{
Transcriptomic analysis implicates necroptosis in disease progression and prognosis in myelodysplastic syndromes
}

\author{
Guillermo Montalban-Bravo ${ }^{1,{ }^{*}, \text { Caleb A. Class }}{ }^{2}$, Irene Ganan-Gomez ${ }^{1}$, Rashmi Kanagal- \\ Shamanna ${ }^{3}$, Koji Sasaki ${ }^{1}$, Guillaume Richard-Carpentier ${ }^{1}$, Kiran Naqvi ${ }^{1}$, Yue Wei ${ }^{1}$, Hui \\ Yang $^{1}$, Kelly A. Soltysiak ${ }^{1}$, Kelly Chien ${ }^{1}$, Carlos Bueso-Ramos ${ }^{3}$, Kim-Anh Do ${ }^{2}$, Hagop \\ Kantarjian ${ }^{1}$, Guillermo Garcia-Manero ${ }^{1}$ \\ ${ }^{1}$ Department of Leukemia, The University of Texas MD Anderson Cancer Center, Houston, TX \\ ${ }^{2}$ Department of Biostatistics, The University of Texas MD Anderson Cancer Center, Houston, TX \\ ${ }^{3}$ Department of Hematopathology, The University of Texas MD Anderson Cancer Center, \\ Houston, TX
}

\begin{abstract}
Myelodysplastic syndromes (MDS) are characterized by ineffective hematopoiesis and cytopenias due to uncontrolled programmed cell death. The presence of pro-inflammatory cytokines and constitutive activation of innate immunity signals in MDS cells suggest inflammatory cell death, such as necroptosis, may be responsible for disease phenotype. We evaluated 64 bone marrow samples from 55 patients with MDS or chronic myelomonocytic leukemia (CMML) obtained prior to $(n=46)$ or after $(n=18)$ therapy with hypomethylating agents (HMAs). RNA from sorted bone marrow CD34+ cells was isolated and subject to amplification and RNA-Seq. Compared to healthy controls, expression levels of $M L K L$ (CMML: $2.09 \log 2 \mathrm{FC}, \mathrm{p}=0.0013$; MDS: 1.89 $\log 2 \mathrm{FC}, \mathrm{p}=0.003$ ), but not $R I P K 1$ or $R I P K 3$, were significantly upregulated. Higher expression levels of $M L K L$ were associated with lower hemoglobin levels at diagnosis $(-0.19 \log 2 \mathrm{FC}$ per
\end{abstract}

Users may view, print, copy, and download text and data-mine the content in such documents, for the purposes of academic research, subject always to the full Conditions of use:http://www.nature.com/authors/editorial_policies/license.html\#terms

*Corresponding author: Guillermo Montalban-Bravo, MD, Department of Leukemia, University of Texas, MD Anderson Cancer Center, 1515 Holcombe Blvd, Unit 428, Houston, TX 77030, Fax: 713-745-4612, Phone: 713-794-3604,

gmontalban1@mdanderson.org.

Competing Interests: The authors declare no competing interests.

Disclosures

Guillermo Montalban-Bravo: This author declares no conflict of interest.

Caleb A. Class: This author declares no conflict of interest.

Irene Ganan-Gomez: This author declares no conflict of interest.

Rashmi Kanagal-Shamanna: This author declares no conflict of interest.

Koji Sasaki: This author declares an advisory role with Pfizer Japan.

Guillaume Richard-Carpentier: This author declares no conflict of interest.

Kiran Naqvi: This author declares no conflict of interest.

Yue Wei: This author declares no conflict of interest.

Hui Yang: This author declares no conflict of interest.

Kelly A. Soltysiak: This author declares no conflict of interest.

Kelly Chien: This author declares no conflict of interest.

Carlos Bueso-Ramos: This author declares no conflict of interest.

Kim-Anh Do: This author declares no conflict of interest.

Data availability statement: The datasets generated during and/or analysed during the current study are not publicly available due to patient privacy concerns but are available from the corresponding author on reasonable request. 
$1 \mathrm{~g} / \mathrm{dL}$ increase of $\mathrm{Hgb}, \mathrm{p}=0.03)$. Significant reduction in $M L K L$ levels was observed after HMA therapy $(-1.06 \log 2 \mathrm{FC}, \mathrm{p}=0.05)$ particularly among non-responders $(-2.89 \log 2 \mathrm{FC}, \mathrm{p}=0.06)$. Higher RIPK1 expression was associated with shorter survival (HR 1.92, 95\% CI 1.00-3.67, $\mathrm{p}=0.049$ by Cox proportional hazards). This data provides further support for a role of necroptosis in MDS, and potentially response to HMAs and prognosis. This data also indicates that RIPK1/ $R I P K 3 / M L K L$ are potential therapeutic targets in MDS.

\section{Keywords}

necroptosis; myelodysplastic syndromes; transcriptomic analysis

\section{INTRODUCTION}

Myelodysplastic syndromes (MDS) are a heterogenous group of disorders characterized by ineffective hematopoiesis leading to cytopenias and a variable risk of progression and transformation to acute myeloid leukemia (AML) $(1,2)$. Although the mechanisms leading to ineffective hematopoiesis have not been fully elucidated, differentiation block and programmed cell death are known contributors to this phenomenon (3). Studies evaluating the role of apoptosis and the expression of apoptosis regulatory genes in MDS (4-8) have led to inconsistent results, with reported rates of apoptosis ranging from 2.5 to $56.3 \%$ and caspase 3, caspase 8, and annexin $\mathrm{V}$ positivity in bone marrow CD34+ cells varying in different studies based on morphologic and prognostic disease subgroups. Overexpression of Fas and Fas-Ligand have been reported in bone marrow CD34+ MDS cells (4), but activation of these receptors could also lead to necrotic cell death (9), and not only apoptosis. In addition, programmed cell death in MDS CD34+ cells has been shown to be upregulated and modulated by Toll-like receptors (TLRs) $(8,10)$. Innate immunity signaling mediated through TLRs, inflammatory cytokines (i.e., IL-1, IL-6, and IL-8), and damage associated molecular patterns (DAMPs) such as S100A8/9 are observed among most MDS subtypes $(10,11)$. This inflammatory background, the unclear role of apoptosis in MDS pathogenesis, and the high levels of tumor necrosis factor (TNF) in MDS (12-14), suggests alternative and less immune-silent mechanisms of cell death may be responsible for the disease phenotype. Although recent studies have described NLRP3-inflammasome mediated pyroptosis as a mediator of the MDS phenotype $(15,16)$, there are limited data evaluating the potential role of other inflammatory mechanisms of cell death in MDS pathogenesis. Recent studies suggest evasion of apoptosis through BCL2 upregulation may be involved in disease progression $(17,18)$. Whether this is the case with other forms of programmed cell death is unknown.

Necroptosis is a form of regulated necrotic cell death that can be activated under apoptosisdeficient conditions and triggered by activation of death receptors (DRs) such as TNF receptor 1 (TNFR1) (9). Under specific conditions, activation of TNFR1 leads to activation of receptor-interacting protein kinase 1 (RIPK1) which phosphorylates and activates RIPK3 to form a RIPK1-RIPK3-mixeld lineage kinase domain-like protein (MLKL) complex responsible for inducing cell death through membrane pore formation. Although necroptosis has been associated with multiple inflammatory conditions $(9,19)$, its potential role in MDS 
remains unclear. A recent study by Wagner, et al evaluated a Vav-Cre-Bax/Bak/Bid triple knock out mouse model and demonstrated that unrestrained bone marrow necroptosis promoted inflammatory cytokine production that impaired hematopoietic stem-cell (HSPC) function. RIPK1 has also been shown to regulate hematopoiesis (20-22), mediate inflammation $(9,22)$, maintain chromosomal stability $(23,24)$, and modulate the expression of immune checkpoint molecules such as PD-1 or PD-L1 (23), which are known to be upregulated in MDS after failure to hypomethylating agent (HMA) therapy (25). In addition, inhibition of RIPK1 has been hypothesized as a potential therapeutic mechanism in several malignancies $(26,27)$.

In this study, we use RNA sequencing to analyze the expression level of the necroptosis complex components RIPK1, RIPK3, and $M L K L$ in a total of 55 patients with MDS or chronic myelomonocytic leukemia (CMML), and to correlate this with response to HMA therapy and expression of apoptosis regulatory proteins and immune checkpoint regulators. We observed that $M L K L$ was upregulated in MDS and CMML, and its downregulation was associated with absence of response to HMAs. In addition, we observed an association between necroptosome components and the $B C L 2$ family of proteins and immune checkpoint regulators. Finally, we identified that RIPK1 expression levels predict prognosis of patients with MDS and were associated with innate immunity and pro-inflammatory signaling in MDS bone marrow CD34+ cells.

\section{MATERIALS AND METHODS}

\section{Patients and Samples}

We evaluated 64 bone marrow aspirates from 55 patients with MDS ( $n=34)$ or CMML $(n=21)$ treated at the University of Texas MD Anderson Cancer Center (MDACC). Samples were obtained prior to $(n=46)$ or after $(n=18)$ HMA therapy. BM samples from healthy individuals were obtained from AllCells (Emeryville, CA). Patient characteristics are detailed in Supplemental Table S1. Informed consent was obtained according to protocols approved by the MDACC institutional review board in accordance with the Declaration of Helsinki. Diagnosis was confirmed in the hematopathology laboratory at MDACC by BM evaluation using 2016 WHO criteria. Conventional karyotyping was performed on fresh BM aspirates using standard procedures and reported following ISCN 2013 Nomenclature. Patients were classified using the revised 2016 WHO classification (28). Prognostic risk was calculated using both the International Prognostic Scoring System (IPSS) (2) and the revised IPSS (1). Response assessment was performed following the IWG 2006 criteria (29). On selected cases, immunohistochemical staining was performed on $4 \mu \mathrm{m}$ thick formalin fixed paraffin embedded BM sections using standard procedures as described elsewhere using a mouse monoclonal antibody for BCL2 oncoprotein (Bcl-2/100/D5; 1:50, Leica(30)).

\section{Isolation of BM CD34+ cells}

MDS and CMML bone marrow (BM) aspirates were freshly obtained from patients referred to the Department of Leukemia at MDACC under approved protocols (LAB01-473). BM samples from healthy individuals were obtained from AllCells (Emeryville, CA). CD34+ cells were isolated using the CD34 MicroBead Kit (Miltenyi Biotec, Canada). 


\section{RNA Sequencing Analysis}

RNA from sorted BM CD34+ cells was isolated using the PicoPure RNA isolation kit (Fisher Scientific, Waltham, MA) before RNA amplification and RNA-Seq library construction. Fastq files were mapped to the human genome (build GRCh38) in TopHat2 using the default options (31). Differential gene expression analysis was conducted using DESeq2 in R version 3.4.2 (32). Gene expression was normalized for plotting using the variance-stabilizing transformation implemented in the DESeq2 package. Gene coexpression was evaluated using Spearman correlation for the full data set, and using the Pearson correlation for analyses within subsets (due to small sample sizes). Pathway enrichment analysis was performed using gene set enrichment analysis, with the fgsea library in R (33). Genes were ranked according to their Spearman correlation with the gene of interest, and this ranking was used as the input to fgsea. 10000 gene permutations were used to calculate statistical significance, and a false discovery corrected p-value of 0.05 was required for statistical significance of a gene set.

\section{Targeted Gene Sequencing}

Genomic DNA was extracted from whole bone marrow samples and subject to ampliconbased targeted next-generation sequencing (NGS) evaluating a panel of 81 genes. This analysis was performed within our CLIA-compliant molecular diagnostics laboratory after informed consent (additional details in Supplemental Methods). A total of $250 \mathrm{ng}$ of genomic DNA extracted from whole mononuclear cells from fresh BM aspirate was used for library preparation using HaloPlex chemistry (Agilent Technologies, Santa Clara, CA). Multiplexed bi-directional sequencing was performed on a MiSeq V3 300 cycle kit (Illumina, SanDiego, CA) using appropriate controls (310 cycles). Sequencing data was processed through MiSeq reporter (Illumina) and SureCall 3.0 (Agilent) with GRCh37/hg 19 as a reference for sequence alignment. For variant calling, a minimum coverage of $250 \mathrm{x}$ in both directions and a minimum of $2 \%$ allelic frequency were required. For confirmation, all variant calls were visualized using Integrative Genomics Viewer (34). Since matched germline controls were not sequenced, the somatic nature of the variants was determined using a combination of parameters. These included variant allele frequencies and available data in the literature and COSMIC. Single nucleotide polymorphisms (SNPs) reported in the Exome Aggregation Consortium (ExAC), dbSNP 137/138, and 1000 Genomes Project were excluded. FLT3 mutational analysis for internal tandem duplication was also performed by PCR and capillary electrophoresis.

\section{RESULTS}

\section{$M L K L$ is overexpressed in MDS and CMML irrespective of WHO category or genomic context, and is associated with anemia severity}

We compared RIPK1, RIPK3, and MLKL expression levels in patients with MDS and CMML at the time of diagnosis. Compared to healthy controls, $M L K L$ (CMML vs controls: $2.09 \log 2 \mathrm{FC}, \mathrm{p}=0.0013$; MDS vs control: $1.89 \log 2 \mathrm{FC}, \mathrm{p}=0.003$ ), but not RIPK1 or RIPK3, were significantly upregulated in patients with MDS and CMML (Figure 1). A trend of higher $M L K L$ and $R I P K 3$ levels was observed in patients with MDS and multilineage dysplasia $(\mathrm{n}=2)$ compared to other MDS subtypes. Targeted sequencing was available at the 
time of diagnosis in 16 patients with MDS and 13 with CMML (Figure S1). We correlated expression levels of $M L K L, R I P K 1$, and RIPK3 with detected mutations. To minimize the risk of biased associations due to small numbers, only mutations observed in at least 3 patients were considered. No differences in the level of expression of RIPK1, MLKL or RIPK 3 were observed based on the mutation context or burden. Since RIPK1 is known to participate in chromosomal stability, we correlated its expression levels with presence of cytogenetic abnormalities. No significant difference in RIPK1, RIPK3 or $M L K L$ expression levels was observed based on presence of cytogenetic abnormalities (RIPK1: $0.10 \log 2 \mathrm{FC}$, $\mathrm{p}=0.6$; RIPK3: $-0.39 \log 2 \mathrm{FC}, \mathrm{p}=0.40$; MLKL: $0.34 \log 2 \mathrm{FC}, \mathrm{p}=0.30)$. In order to determine whether higher expression levels of $M L K L$ translated into worse ineffective hematopoiesis, we correlated $M L K L$ expression with the number and degree of cytopenias. Higher expression levels of $M L K L$ were associated with lower hemoglobin levels at the time of diagnosis $(-0.19 \log 2 \mathrm{FC}$ per $1 \mathrm{~g} / \mathrm{dL}$ increase of $\mathrm{Hgb}, \mathrm{p}=0.03$ ) (Figure 1). Since MLKLmediated necroptosis is associated with cytokine release and a pro-inflammatory response, and given the association between $M L K L$ expression and anemia, we evaluated the association between $M L K L$ expression and cytokine signaling. $M L K L$ expression was significantly correlated with cytokine signaling using Gene Set Enrichment Analysis (NES 2.51, $\mathrm{q}=0.024$ ) (Figures 1 and S2). We then associated levels of expression of receptors associated with induction of necroptosis. Compared to healthy controls, expression levels of TRAIL, but not TNFRSF1A, were significantly higher in patients with CMML and MDS (CMML vs control: $3.22 \log 2 \mathrm{FC}, \mathrm{p}<0.001, \mathrm{q}<0.001$; MDS vs control: $2.59 \log 2 \mathrm{FC}$, $\mathrm{p}<0.001, \mathrm{q}<0.001)$.

\section{$M L K L$ is downregulated in bone marrow CD34+ cells from patients with MDS who do not achieve response to azacitidine or decitabine}

In order to assess whether HMA treatment modified the expression profile of the necroptosis complex, we evaluated RIPK1, RIPK3, and MLKL levels after HMA therapy in patient matched and unmatched samples. Clinical characteristics of responders and non-responders are detailed in Supplemental Table S2. As observed prior to therapy, $M L K L$ levels, but not RIKP1 and RIKP3, were significantly increased both in MDS and CMML compared to healthy controls (Figure S3). Exposure to HMA therapy was associated with a trend to decreased expression of $M L K L(-0.52 \log 2 \mathrm{FC}, \mathrm{p}=0.08)$ when all post-HMA therapy samples were evaluated. Among patient matched samples, a significant reduction in $M L K L$ levels was observed after HMA therapy $(-1.06 \log 2 \mathrm{FC}, \mathrm{p}=0.05)$. The degree of reduction in expression levels was greater among non-responders $(-2.89 \log 2 \mathrm{FC}, \mathrm{p}=0.06)$ compared to patients who experienced a response to therapy $(-0.78, \log 2 \mathrm{FC}, \mathrm{p}=0.06)$. Progression to AML with expansion of the blast population was observed in the 2 non-responder patients with matched pre- and post-therapy samples.

\section{RIPK1 and MLKL expression levels correlate with expression of the BCL2 family of proteins and $P D-1$ after exposure to HMAs}

Because necroptosis can only occur in apoptosis-deficient conditions and is associated with immune regulation, we studied the correlation of RIPK1, RIPK3, and $M L K L$ expression levels with those of the BCL2 family of proteins and immune checkpoint regulatory molecules PD-1, PD-L1, and CTLA4. Levels of RIPK3 were negatively correlated with 
$B A K$ and positively correlated with $B I D$ and $B I M$ in responding patients prior to therapy $(\mathrm{n}=16)$ and strongly correlated with $P D-1$ and negatively correlated with $B I M$ after therapy $(\mathrm{n}=7)$ (Figure 2). In addition, $M L K L$ expression was strongly correlated with $B C L 2$ expression. Among non-responders, RIPK1 and $R I P K 3$ expression was strongly correlated with $P U M A$ and $B A K$ prior to therapy (n=11) (Figure 2) with $M L K L$ expression correlating with $B I D$ and $P U M A$ levels, RIPK3 with $P D-1$ levels and RIPK1 with $B A K$ after therapy $(\mathrm{n}=6)$. In addition, $R I P K 1$ had a strong negative correlation with $B A X$ levels.

In view of the overexpression of $M L K L$ compared to healthy controls, the decrease in expression levels after HMA therapy, and their correlation with BCL2 expression, we evaluated BCL2 protein expression in blasts/immature cells by IHC in a subset $(\mathrm{n}=11)$ of patients before $(\mathrm{n}=8)$ and after therapy $(\mathrm{n}=3)$ with HMA (Table 1$)$. Among pre-treatment samples from responders $(\mathrm{n}=4), M L K L$ was overexpressed in all compared to controls. No significant BCL2 protein expression could be observed in blasts/immature cells. In addition, $B C L 2$ mRNA expression was decreased compared to controls in 3 patients. Among pretreatment samples from non-responders $(n=4), B C L 2$ protein detection by IHC was strongly positive in 2 patients coinciding with high $B C L 2$ RNA expression, and negative in 2 patients with decreased BCL2 RNA expression. $M L K L$ was overexpressed compared to controls in all 4 patients. Finally, post-treatment samples from non-responders $(\mathrm{n}=3)$ revealed BCL2 IHC positivity in all patients, along with downregulation of $M L K L$ and upregulation of $B C L 2$ in 2 patients. Examples of positive and negative BCL2 IHC are shown in Supplemental Figure S4.

\section{RIPK1 levels are associated with adverse prognosis and distinct gene pathway enrichment}

We next evaluated the impact of RIPK1, RIPK3, and $M L K L$ expression levels on prognosis of patients with MDS and CMML. Expression levels of RIPK1 at the time of diagnosis predicted for shorter survival for patients with high RIPK1 levels, defined as a log expression higher than median mRNA expression values, having significantly shorter overall survival compared to those with lower expression levels (median OS 10.7 vs 24.2 months, HR 1.92, 95\% CI 1.00-3.67, $\mathrm{p}=0.049$ by Cox proportional hazards) (Figure 3). This survival difference was even more notable when evaluating MDS patients only (median OS 10.5 months vs 24.2 months, HR 7.09, 95\% CI 2.06-24.3, $\mathrm{p}=0.002$ by Cox proportional hazards) (Figure 3). Although patients with higher RIPK1 levels showed a trend toward have higher bone marrow blast percentage, higher IPSS-R risk categories, and lower hemoglobin and platelets, these differences were not significant (Supplemental Figure S5). Distribution of WHO subtype, IPSS-R risk category, cytogenetics, and mutation profile based on RIPK1 expression is shown in Figure 4. A multivariate analysis for overall survival using both IPSS-R risk and RIPK1 expression levels demonstrated that high RIPK1 expression was an independent adverse prognostic factor in MDS patients (HR 6.83, 95\% CI 1.74-26.8, $\mathrm{p}=0.006$ ).

To elucidate the potential mechanisms underlying this survival difference we evaluated differentially expressed genes in patients with high compared to low RIPK1 expression. A total of 359 genes were significantly correlated with RIPK1 levels in MDS CD34+ cells (Spearman's method q $<0.2$, Supplemental Table S3). Among these, 21 genes were positively 
correlated with RIPK1 expression, while 17 genes were negative correlated (Spearman's correlation, $\mathrm{q}<0.1$ ). Gene set enrichment analysis identified 373 gene sets that significantly correlated with RIPK1 expression (Supplemental Table S4). Upregulated genes were associated with molecular signals associated with innate immunity and inflammatory signaling including TNF-a signaling via NFkB, NFkB signaling, the IFN- $\gamma$ pathway, and RIG-I-like receptor (RLRs) signaling genes (Figure 5). Genes found to be differentially downregulated were associated with ATP synthesis and respiratory electron transport, oxidative phosphorylation, APC/CDC20 mediated degradation of mitotic proteins, and E2F targets.

\section{DISCUSSION}

Increasing evidence supports the involvement of inflammatory forms of cell death, such as pyroptosis, in MDS pathogenesis (15). Although a recent study suggests the potential role of necroptosis in MDS pathophysiology (35), the implications of this inflammatory cell death in disease progression, response to therapy or prognosis are unknown. In this study, we performed RNA-Seq on isolated CD34+ bone marrow cells from patients with MDS and CMML, and observed overexpression of $M L K L$, the effector molecule of necroptosis, and determined its association with the severity of anemia and response to hypomethylating agents. In addition, we determined that necroptosome component expression may be associated with expression of the BCL2 family of proteins and immune checkpoint regulators and that RIPK1 expression levels were associated with worse than expected survival, as determined by IPSS-R, and enriched innate immunity and inflammatory signaling.

Ineffective hematopoiesis in MDS has been traditionally associated with impaired differentiation and apoptosis of hematopoietic stem and progenitor cells (HSPCs). Recent studies suggest the potential role of inflammatory forms of death in MDS. In a study by Basiorka, et al (16), NRLP3-inflammasome mediated pyroptosis was found to participate and be particularly enriched compared to apoptosis in patients with lower-risk MDS. More recently, Wagner, et al (35) reported bone marrow failure and myelodysplasia in a triple knock out ( $\mathrm{Vav}$-Cre Bax/Bak/Bid), apoptosis-resistant mouse model in which increased RIPK1 kinase activity mediated bone marrow necroptosis. Increased expression of RIPK1 and pMLKL by immunofluorescence was also observed in bone marrow samples from patients with MDS, particularly among those with MDS with multilineage dysplasia (MDSMLD) compared to those with excess blasts (MDS-EB). This data suggests necroptosis may be responsible for early stages of the disease and that, as the disease progresses, additional genomic and epigenomic abnormalities may lead to evasion of cell death and clonal expansion. In our study, by evaluating necroptosome components at the transcriptomic level, we also identified overexpression of $M L K L$ in MDS and a trend toward higher expression in patients with MDS-MLD. In addition, higher expression levels were associated with more severe anemia, which supports the notion that necroptosis may mediate ineffective erythropoiesis. Future studies confirming whether increased $M L K L$ expression leads to impaired erythroid colony formation will be required. Also, although increased expression levels of RIPK1 at the time of diagnosis independently predicted for a worse overall survival, the underlying mechanism behind this remains unclear. Although Wagner et al 
demonstrated that samples from patients with MDS-MLD and MDS with ring sideroblasts showed increased expression of RIPK1 compared to 50\% of samples from patients with MDS-EB, we did not observe significant differences in RIPK1 expression based on MDS WHO subtype, blast percentage, IPSS or IPSS-R category. This may be related to small patient numbers (22 samples in the study by Wagner, et al and 64 in our study) or differences in the study populations, such as a higher proportion of MDS-EB in our study. Further confirmation of the degree of activity and transcription of RIPK1 will be needed. By evaluating necroptosome component expression after therapy with the HMAs azacitidine and decitabine, we observed significant downregulation of $M L K L$, particularly among nonresponding patients who also experienced progressive disease with blast expansion at the time of failure. This suggests that, during the process of emergence of resistance to HMAs, HSPCs may downregulate $M L K L$ to evade necroptosis and have a survival advantage facilitating clonal expansion and disease progression. Similar functional changes leading to evasion of apoptosis have been described at the time of failure to HMA due to upregulation of $B C L 2(17,18)$. In our study, we observed a strong correlation between $M L K L, B I M$, and $P U M A$ expression after therapy in non-responding patients, further suggesting that evasion of apoptosis and necroptosis may be involved in disease progression. By evaluating BCL2 protein expression by IHC, we observed that only non-responding patients had detectable BCL2 protein in blasts and immature myeloid cells, particularly after therapy with HMA, and this was associated with corresponding upregulation of $B C L 2$ at the RNA level along with downregulation of $M L K L$ RNA compared to pre-treatment in 2 out of 3 patients. Future functional studies will be required to evaluate this relationship further and confirm whether downregulation of necroptosis along with upregulation of pro-survival BCL2 family of proteins may be responsible for disease progression.

Upregulation of innate immunity and pro-inflammatory signaling are involved in MDS pathogenesis $(11,36)$. Several studies have shown constitutive TLR signaling in MDS leading to upregulation of genes involved in NFKB signaling, IL-8 over-production, and inhibition of colony formation potential, particularly in CD34+ cells from patients with previous exposure to HMAs $(10,37)$. In our cohort, higher expression levels of $R I P K 1$ were associated with significant overexpression of genes involved in TNF- $a$ and NFKB signaling and RLRs, which mediate PAMP recognition, immune signaling, and production of IFN (38). In line with previous studies $(22,23)$, this data further supports the role of RIPK1 as a mediator of inflammation. Given that in our study, high RIPK1 expression was an independent adverse prognostic factor in patients with MDS and was associated with upregulation of innate immunity signaling, inhibition of RIPK1 alone or in combination with drugs targeting inflammation or innate immunity signaling might have a therapeutic role in MDS. In fact, we have previously reported the therapeutic potential associated with targeting innate immunity signaling in MDS by inhibition of JAK/STAT (39), TLR2 (40), p38 MAPK (41), or NFKB $(40,42)$. Moreover, consistent with previous studies linking RIPK1 function with PD-1 expression (23), we observed an association between RIPK3 and PD-1 expression levels. Given the preclinical data suggesting RIPK1 inhibition leads to upregulation of PD-1 (23), the fact that overexpression of PD-1 may lead to ineffective hematopoiesis (43) and potentially to HMA failure (25) and the clinical activity of PD-1 inhibitors in MDS (44), this could support the concept of exploring dual RIPK1 and PD-1 inhibition in MDS. 
We acknowledge our study has several limitations. First, although mRNA expression is a valid way to assess the degree of activity of a given biological function, it is not an optimal tool to evaluate kinase activity and absence of phosphorylated protein evaluation limits our ability to confirm protein activation and degree of expression. In addition, the patient numbers included in our study are small and therefore limit the statistical power needed to generate robust associations. Third, only a subset of included samples corresponded to matched pre and post therapy samples, which this limits the ability to conclude whether the observed changes in expression are specific to therapy. Finally, functional studies will be required to confirm our findings and larger studies are necessary to evaluate the role and the prognostic significance of necroptosis in MDS and the functional link between evasion of necroptosis and disease progression.

Despite these limitations, our study is consistent with prior studies suggesting the potential role of necroptosis in MDS. In addition, to the best of our knowledge, this is the first study to associate expression of necroptosome components with loss of response and progression after HMA therapy in patients with MDS as well as prognosis, PD-1, and innate immunity expression. This sets the basis for future studies confirming these findings and evaluating RIPK1/RIPK3/MLKL as therapeutic targets for MDS alone or in combination with PD-1 monoclonal antibodies or novel agents targeting innate immunity or inflammatory signaling.

\section{Supplementary Material}

Refer to Web version on PubMed Central for supplementary material.

\section{Acknowledgements}

This work was supported in part by the University of Texas MD Anderson Cancer Center Support Grant CA016672 and the University of Texas MD Anderson MDS/AML Moon Shot.

Hagop Kantarjian: This author declares research support and an advisory role with Actinium, and research support from AbbVie, Agio, Amgen, Ariad, Astex, BMS, Cyclacel, Daiichi-Sankyo, Immunogen, Jazz Pharma, Novartis, and Pfizer.

Guillermo Garcia-Manero: This author declares research support and an advisory role with Amphivena, Astex, and Celgene, and research support from AbbVie, H3 Biomedicine, Helsin, Onconova, Merck, and Novartis.

\section{REFERENCES}

1. Greenberg PL, Tuechler H, Schanz J, Sanz G, Garcia-Manero G, Sole F, et al. Revised international prognostic scoring system for myelodysplastic syndromes. Blood. 2012;120(12):2454-65. [PubMed: 22740453]

2. Greenberg P, Cox C, LeBeau MM, Fenaux P, Morel P, Sanz G, et al. International scoring system for evaluating prognosis in myelodysplastic syndromes. Blood. 1997;89(6):2079-88. [PubMed: 9058730]

3. Schneider RK, Schenone M, Ferreira MV, Kramann R, Joyce CE, Hartigan C, et al. Rps14 haploinsufficiency causes a block in erythroid differentiation mediated by S100A8 and S100A9. Nat Med. 2016;22(3):288-97. [PubMed: 26878232]

4. Economopoulou C, Pappa V, Kontsioti F, Papageorgiou S, Kapsimali V, Papasteriadi C, et al. Analysis of apoptosis regulatory genes expression in the bone marrow (BM) of adult de novo myelodysplastic syndromes (MDS). Leuk Res. 2008;32(1):61-9. [PubMed: 17597205] 
5. Economopoulou C, Pappa V, Papageorgiou S, Kontsioti F, Economopoulou P, Charitidou E, et al. Cell cycle and apoptosis regulatory gene expression in the bone marrow of patients with de novo myelodysplastic syndromes (MDS). Ann Hematol. 2010;89(4):349-58. [PubMed: 19813013]

6. Langemeijer SM, Mariani N, Knops R, Gilissen C, Woestenenk R, de Witte T, et al. ApoptosisRelated Gene Expression Profiling in Hematopoietic Cell Fractions of MDS Patients. PLoS One. 2016;11(11):e0165582. [PubMed: 27902785]

7. Suarez L, Vidriales MB, Sanz G, Lopez A, Lopez-Berges MC, de Santiago M, et al. Expression of APO2.7, bcl-2 and bax apoptosis-associated proteins in CD34- bone marrow cell compartments from patients with myelodysplastic syndromes. Leukemia. 2004;18(7):1311-3. [PubMed: 15116120]

8. Zeng Q, Shu J, Hu Q, Zhou SH, Qian YM, Hu MH, et al. Apoptosis in human myelodysplastic syndrome CD34+ cells is modulated by the upregulation of TLRs and histone $\mathrm{H} 4$ acetylation via a beta-arrestin 1 dependent mechanism. Exp Cell Res. 2016;340(1):22-31. [PubMed: 26708616]

9. Yuan J, Amin P, Ofengeim D. Necroptosis and RIPK1-mediated neuroinflammation in CNS diseases. Nat Rev Neurosci. 2019;20(1):19-33. [PubMed: 30467385]

10. Wei Y, Dimicoli S, Bueso-Ramos C, Chen R, Yang H, Neuberg D, et al. Toll-like receptor alterations in myelodysplastic syndrome. Leukemia. 2013;27(9):1832-40. [PubMed: 23765228]

11. Ganan-Gomez I, Wei Y, Starczynowski DT, Colla S, Yang H, Cabrero-Calvo M, et al. Deregulation of innate immune and inflammatory signaling in myelodysplastic syndromes. Leukemia. 2015.

12. Stifter G, Heiss S, Gastl G, Tzankov A, Stauder R. Over-expression of tumor necrosis factor-alpha in bone marrow biopsies from patients with myelodysplastic syndromes: relationship to anemia and prognosis. Eur J Haematol. 2005;75(6):485-91. [PubMed: 16313260]

13. Campioni D, Secchiero P, Corallini F, Melloni E, Capitani S, Lanza F, et al. Evidence for a role of TNF-related apoptosis-inducing ligand (TRAIL) in the anemia of myelodysplastic syndromes. Am J Pathol. 2005;166(2):557-63. [PubMed: 15681838]

14. Zhang Z, Li X, Guo J, Xu F, He Q, Zhao Y, et al. Interleukin-17 enhances the production of interferon-gamma and tumour necrosis factor-alpha by bone marrow $\mathrm{T}$ lymphocytes from patients with lower risk myelodysplastic syndromes. Eur J Haematol. 2013;90(5):375-84. [PubMed: 23331180]

15. Basiorka AA, McGraw KL, Eksioglu EA, Chen X, Johnson J, Zhang L, et al. The NLRP3 inflammasome functions as a driver of the myelodysplastic syndrome phenotype. Blood. 2016;128(25):2960-75. [PubMed: 27737891]

16. Basiorka AA, McGraw KL, Abbas-Aghababazadeh F, McLemore AF, Vincelette ND, Ward GA, et al. Assessment of ASC specks as a putative biomarker of pyroptosis in myelodysplastic syndromes: an observational cohort study. Lancet Haematol. 2018;5(9):e393-e402. [PubMed: 30072146]

17. Ganan-Gomez I, Alfonso A, Yang H, Santoni A, Marchica V, Fiorini E, et al. Cell-Type Specific Mechanisms of Hematopoietic Stem Cell (HSC) Expansion Underpin Progressive Disease in Myelodysplastic Syndromes (MDS) and Provide a Rationale for Targeted Therapies. Blood. 2018;132(Suppl 1):1798-.

18. Vidal V, Robert G, Goursaud L, Durand L, Ginet C, Karsenti JM, et al. BCL2L10 positive cells in bone marrow are an independent prognostic factor of azacitidine outcome in myelodysplastic syndrome and acute myeloid leukemia. Oncotarget. 2017;8(29):47103-9. [PubMed: 28514758]

19. Jang KH, Do YJ, Koo TS, Choi JS, Song EJ, Hwang Y, et al. Protective effect of RIPK1-inhibitory compound in in vivo models for retinal degenerative disease. Exp Eye Res. 2018;180:8-17. [PubMed: 30500363]

20. Annibaldi A, Meier P. Ripk1 and haematopoiesis: a case for LUBAC and Ripk3. Cell Death Differ. 2018;25(8):1361-3. [PubMed: 29867129]

21. Peltzer N, Darding M, Montinaro A, Draber P, Draberova H, Kupka S, et al. LUBAC is essential for embryogenesis by preventing cell death and enabling haematopoiesis. Nature. 2018;557(7703):112-7. [PubMed: 29695863]

22. Rickard JA, O’Donnell JA, Evans JM, Lalaoui N, Poh AR, Rogers T, et al. RIPK1 regulates RIPK3-MLKL-driven systemic inflammation and emergency hematopoiesis. Cell. 2014;157(5):1175-88. [PubMed: 24813849] 
23. Wang W, Marinis JM, Beal AM, Savadkar S, Wu Y, Khan M, et al. RIP1 Kinase Drives Macrophage-Mediated Adaptive Immune Tolerance in Pancreatic Cancer. Cancer Cell. 2018;34(5):757-74 e7. [PubMed: 30423296]

24. Liccardi G, Ramos Garcia L, Tenev T, Annibaldi A, Legrand AJ, Robertson D, et al. RIPK1 and Caspase-8 Ensure Chromosome Stability Independently of Their Role in Cell Death and Inflammation. Mol Cell. 2019;73(3):413-28 e7. [PubMed: 30598363]

25. Yang H, Bueso-Ramos C, DiNardo C, Estecio MR, Davanlou M, Geng QR, et al. Expression of PD-L1, PD-L2, PD-1 and CTLA4 in myelodysplastic syndromes is enhanced by treatment with hypomethylating agents. Leukemia. 2014;28(6):1280-8. [PubMed: 24270737]

26. Li Y, Xiong Y, Zhang G, Zhang L, Yang W, Yang J, et al. Identification of 5-(2,3-dihydro-1Hindol-5-yl)-7H-pyrrolo[2,3-d]pyrimidin-4-amine Derivatives as a New Class of ReceptorInteracting Protein Kinase 1 (RIPK1) Inhibitors, Which Showed Potent Activity in a Tumor Metastasis Model. J Med Chem. 2018.

27. Huang H, Chen T, Zhou Y, Geng L, Shen T, Zhou L, et al. RIPK1 Inhibition Enhances Pirarubicin Cytotoxic Efficacy through AKT-P21-dependent Pathway in Hepatocellular Carcinoma. Int J Med Sci. 2018;15(14):1648-57. [PubMed: 30588188]

28. Arber DA, Orazi A, Hasserjian R, Thiele J, Borowitz MJ, Le Beau MM, et al. The 2016 revision to the World Health Organization (WHO) classification of myeloid neoplasms and acute leukemia. Blood. 2016.

29. Cheson BD, Bennett JM, Kopecky KJ, Buchner T, Willman CL, Estey EH, et al. Revised recommendations of the International Working Group for Diagnosis, Standardization of Response Criteria, Treatment Outcomes, and Reporting Standards for Therapeutic Trials in Acute Myeloid Leukemia. J Clin Oncol. 2003;21(24):4642-9. [PubMed: 14673054]

30. Kanagal-Shamanna R, Medeiros LJ, Lu G, Wang SA, Manning JT, Lin P, et al. High-grade B cell lymphoma, unclassifiable, with blastoid features: an unusual morphological subgroup associated frequently with BCL2 and/or MYC gene rearrangements and a poor prognosis. Histopathology. 2012;61(5):945-54. [PubMed: 22804688]

31. Kim D, Pertea G, Trapnell C, Pimentel H, Kelley R, Salzberg SL. TopHat2: accurate alignment of transcriptomes in the presence of insertions, deletions and gene fusions. Genome Biol. 2013;14(4):R36. [PubMed: 23618408]

32. Love MI, Huber W, Anders S. Moderated estimation of fold change and dispersion for RNA-seq data with DESeq2. Genome Biol. 2014;15(12):550. [PubMed: 25516281]

33. Subramanian A, Tamayo P, Mootha VK, Mukherjee S, Ebert BL, Gillette MA, et al. Gene set enrichment analysis: a knowledge-based approach for interpreting genome-wide expression profiles. Proc Natl Acad Sci U S A. 2005;102(43):15545-50. [PubMed: 16199517]

34. Pronk CJ, Rossi DJ, Mansson R, Attema JL, Norddahl GL, Chan CK, et al. Elucidation of the phenotypic, functional, and molecular topography of a myeloerythroid progenitor cell hierarchy. Cell Stem Cell. 2007;1(4):428-42. [PubMed: 18371379]

35. Wagner PN, Shi Q, Salisbury-Ruf CT, Zou J, Savona MR, Fedoriw Y, et al. Increased Ripk1mediated bone marrow necroptosis leads to myelodysplasia and bone marrow failure in mice. Blood. 2019;133(2):107-20. [PubMed: 30413413]

36. Wei Y, Zheng H, Bao N, Jiang S, Bueso-Ramos CE, Khoury J, et al. KDM6B overexpression activates innate immune signaling and impairs hematopoiesis in mice. Blood Adv. 2018;2(19):2491-504. [PubMed: 30275007]

37. Wei Y, Chen R, Dimicoli S, Bueso-Ramos C, Neuberg D, Pierce S, et al. Global H3K4me3 genome mapping reveals alterations of innate immunity signaling and overexpression of JMJD3 in human myelodysplastic syndrome CD34+ cells. Leukemia. 2013;27(11):2177-86. [PubMed: 23538751]

38. Loo YM, Gale M, Jr. Immune signaling by RIG-I-like receptors. Immunity. 2011;34(5):680-92. [PubMed: 21616437]

39. Abaza Y, Hidalgo-Lopez JE, Verstovsek S, Jabbour E, Ravandi F, Borthakur G, et al. Phase I study of ruxolitinib in previously treated patients with low or intermediate-1 risk myelodysplastic syndrome with evidence of NF-kB activation. Leuk Res. 2018;73:78-85. [PubMed: 30245189]

40. Garcia-Manero G, Jabbour EJ, Konopleva MY, Daver NG, Borthakur G, DiNardo CD, et al. A Clinical Study of Tomaralimab (OPN-305), a Toll-like Receptor 2 (TLR-2) Antibody, in Heavily 
Pre-Treated Transfusion Dependent Patients with Lower Risk Myelodysplastic Syndromes (MDS) That Have Received and Failed on Prior Hypomethylating Agent (HMA) Therapy. Blood. 2018;132(Suppl 1):798-.

41. Garcia-Manero G, Khoury HJ, Jabbour E, Lancet J, Winski SL, Cable L, et al. A phase I study of oral ARRY-614, a p38 MAPK/Tie2 dual inhibitor, in patients with low or intermediate-1 risk myelodysplastic syndromes. Clin Cancer Res. 2015;21(5):985-94. [PubMed: 25480830]

42. Daher M, Hidalgo Lopez JE, Randhawa JK, Jabbar KJ, Wei Y, Pemmaraju N, et al. An exploratory clinical trial of bortezomib in patients with lower risk myelodysplastic syndromes. Am J Hematol. 2017;92(7):674-82. [PubMed: 28370157]

43. Cheng P, Eksioglu EA, Chen X, Kandell W, Le Trinh T, Cen L, et al. S100A9-induced overexpression of PD-1/PD-L1 contributes to ineffective hematopoiesis in myelodysplastic syndromes. Leukemia. 2019.

44. Garcia-Manero G, Sasaki K, Montalban-Bravo G, Daver NG, Jabbour EJ, Alvarado Y, et al. A Phase II Study of Nivolumab or Ipilimumab with or without Azacitidine for Patients with Myelodysplastic Syndrome (MDS). Blood. 2018;132(Suppl 1):465-. [PubMed: 30072414] 
A

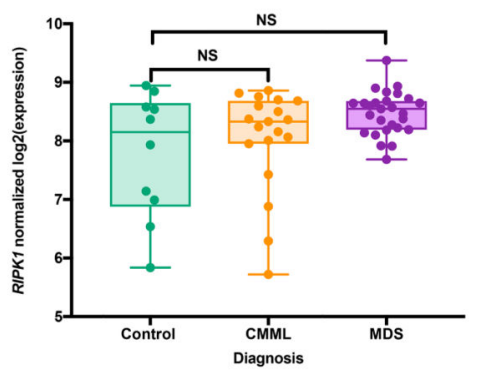

D

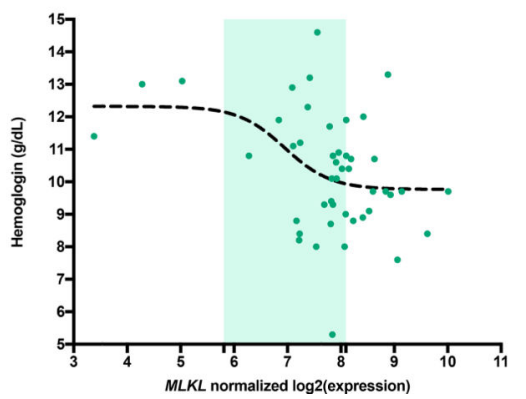

B

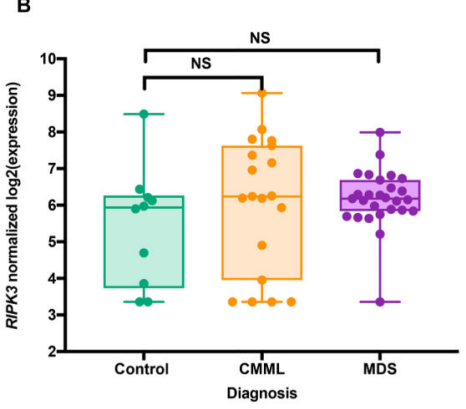

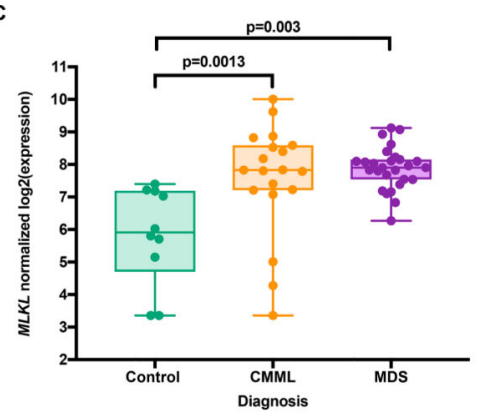

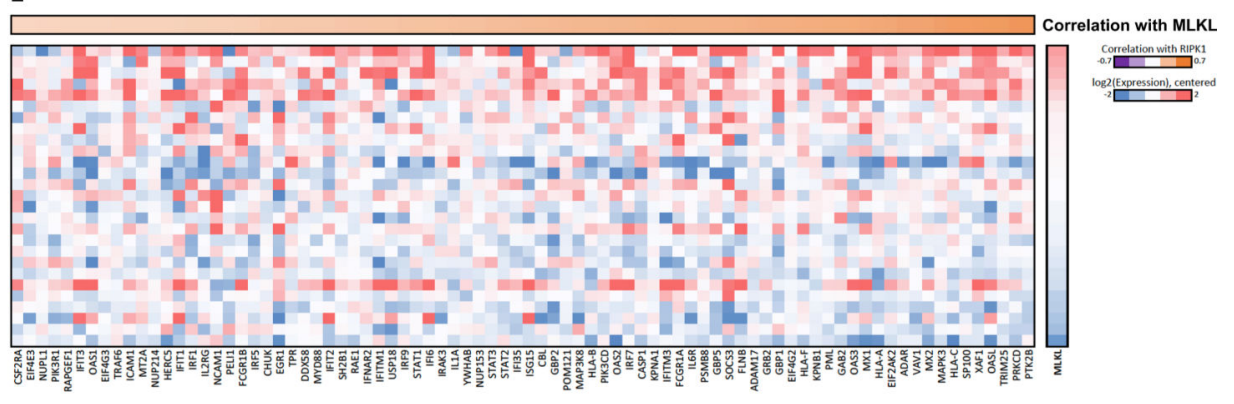

Figure 1. mRNA expression levels of necroptosome components in MDS and CMML and its association with cytopenias.

(A-C) Normalized $\log 2$ mRNA expression levels of RIPK1, RIPK3, and $M L K L$ in bone marrow CD34+ cells from healthy controls, patients with myelodysplastic syndromes (MDS), or chronic myelomonocytic leukemia (CMML). Gene expression was normalized for plotting using the variance-stabilizing transformation implemented in the DESeq2 package. (D) Correlation of normalized $M L K L \log 2$ mRNA expression levels with hemoglobin $(\mathrm{g} / \mathrm{dL})$. (E) Gene set enrichment analysis showing correlation between $M L K L$ expression levels and cytokine signaling genes. Heatmap shows degree of correlation and $\log 2$ expression of each given gene. 
A

B

RIPK1

RIPK3

$M L K L$

Pre-treat ment non-responders $(n=11)$

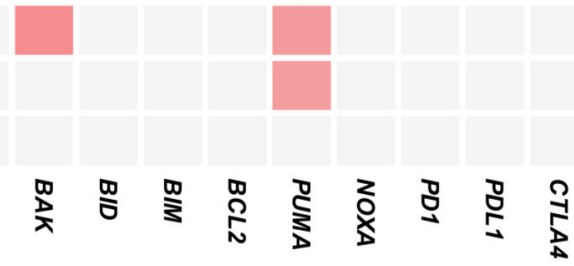

Pre-treatment responders $(n=16)$

RIPK1

RIPK3

$M L K L$

$$
\text { 䍗 界 界 }
$$

RIPK1

RIPK3

$M L K L$

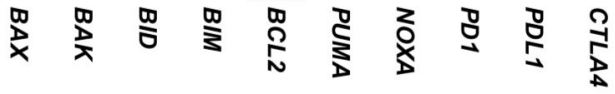

Post-treatment non-responders $(n=6)$

RIPK1

RIPK3

$M L K L$

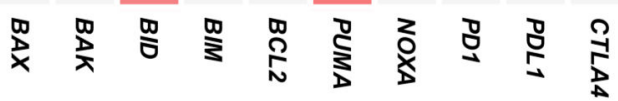

Pearson correlation:

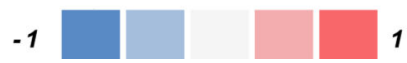

Figure 2. Association of necroptosome component expression levels with the BCL2 family of proteins and immune checkpoint regulators.

Graphs represent correlograms of $R I P K 1, R I P K 3$, and $M L K L$ expression levels from bone marrow CD34+ cells with those of the BCL2 family of proteins and immune checkpoint regulatory molecules $P D-1, P D-L 1$, and $C T L A 4$. Only statistically significant associations ( $\mathrm{p}<0.05)$ are represented. (A) Levels of $R I P K 3$ were negatively correlated with $B A K$ and positively correlated with $B I D$ and $B I M$ in responding patients prior to therapy and strongly correlated with $P D-1$ and negatively correlated with $B I M$ after therapy. In addition, $M L K L$ expression was strongly correlated with $B C L 2$ expression. (B) Among non-responders, $R I P K 1$ and $R I P K 3$ expression was strongly correlated with $P U M A$ and $B A K$ prior to therapy with $M L K L$ expression correlating with $B I D$ and $P U M A$ levels, RIPK3 with $P D-1$ levels and $R I P K 1$ with $B A K$ after therapy. In addition, $R I P K 1$ had a strong negative correlation with $B A X$ levels. 
A

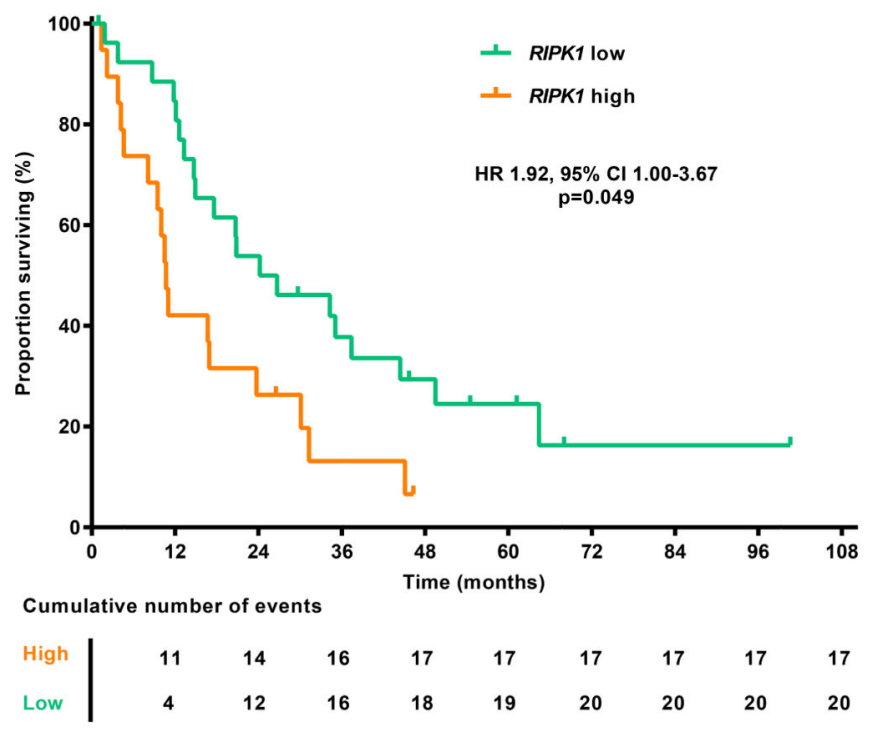

B

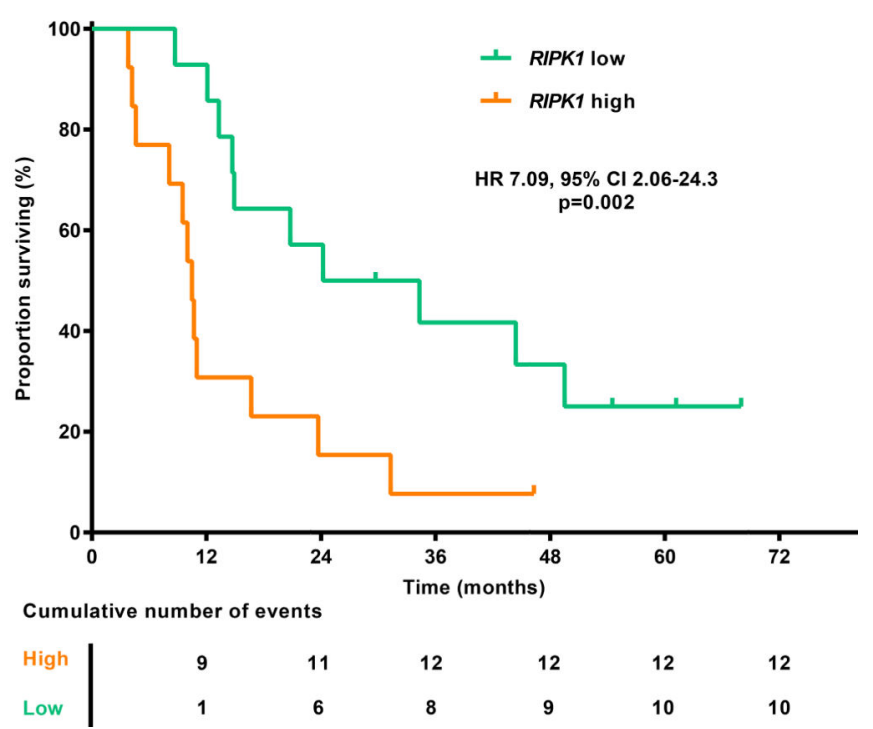

Figure 3. Impact of RIPK1 expression levels on survival of patients with MDS and CMML.

(A) Kaplan-Meier survival estimate curve for overall survival based on high $(\log 2 \mathrm{FC}$ expression above median) versus low (log2FC expression below median) RIPK1 mRNA expression levels in bone marrow CD34+ cells from patients with MDS and CMML. (B) Kaplan-Meier survival estimate curve for overall survival based on high $(\log 2 \mathrm{FC}$ expression above median) versus low (log2FC expression below median) RIPK1 mRNA expression levels in patients with MDS. 


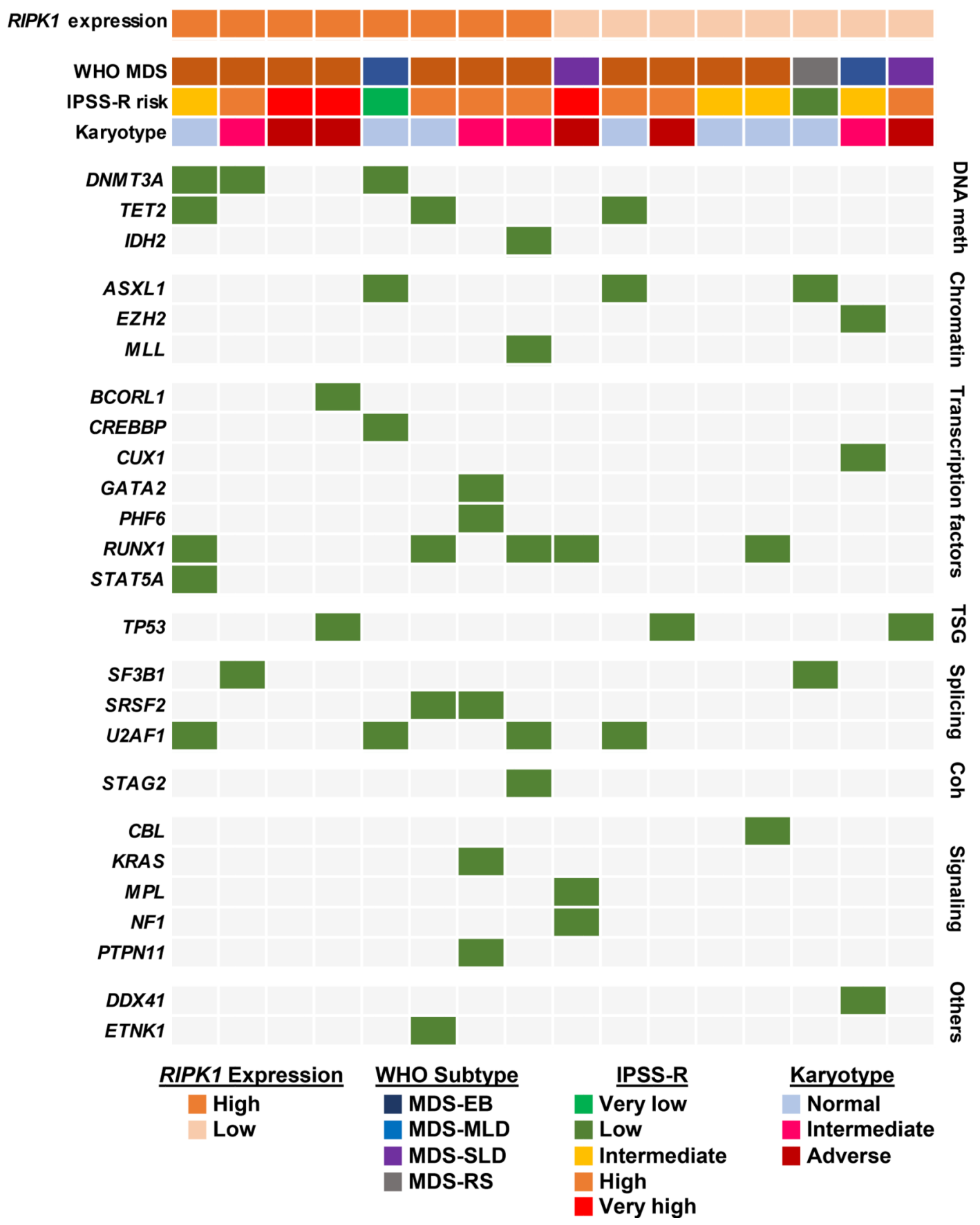

Figure 4. Clinical and genomic features of MDS patients based on RIPK1 expression level. Heat map details the WHO category, IPSS-R category, karyotype, and NGS mutational profiles of patients with MDS at the time of diagnosis. Patient population is clustered based on RIPK1 expression levels: high (mRNA expression levels >median mRNA expression levels) or low (mRNA expression levels <median mRNA expression levels). DNA meth: DNA methylation. TSG: tumor suppressor genes. Coh: cohesins. 


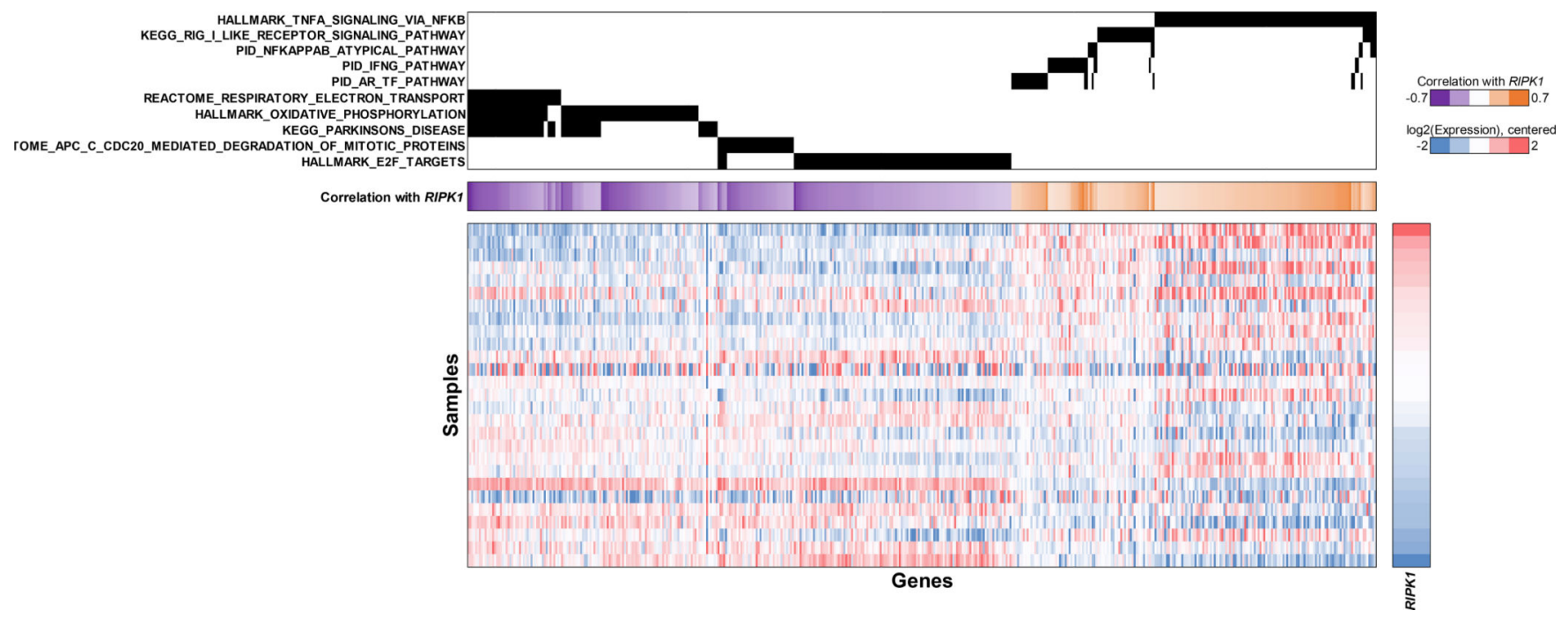

Figure 5. Genomic set enrichment analysis showing differentially regulated pathways in patients with high RIPK1 expression.

Differentially upregulated and downregulated genes and pathways based on gene set enrichment analysis of patients with high RIPK1 expression levels. Significantly enriched pathways (FDR-adjusted $\mathrm{p}<0.05$ ) were ranked by normalized enrichment score, and the top pathways in both directions are presented. Upregulated genes are associated with innate immunity and inflammatory signaling including TNF-a signaling via $\mathrm{NF} \kappa \mathrm{B}, \mathrm{NF} \kappa \mathrm{B}$ signaling, IFN- $\gamma$ pathway, and RIG-I-like receptor signaling genes. Downregulated genes are associated with ATP synthesis and respiratory electron transport, oxidative phosphorylation, APC/CDC20 mediated degradation of mitotic proteins, and E2F targets. 
Table 1.

BCL2 protein expression by immunohistochemistry and its association with $M L K L$ and $B C L 2$ RNA expression levels in responders and non-responders.

\begin{tabular}{|l|c|c|c|c|c|c|}
\hline Time point & Responder & BCL2 IHC & $\begin{array}{c}\text { MLKL log2FC } \\
\text { expression }\end{array}$ & $\begin{array}{c}\text { MLKL compared to } \\
\text { median }\end{array}$ & $\begin{array}{c}\text { BCL2 log2FC } \\
\text { expression }\end{array}$ & $\begin{array}{c}\text { BCL2 compared to } \\
\text { median }\end{array}$ \\
\hline Pre-treatment & Yes & Negative & 7.23 & Up & 10.06 & Up \\
\hline Pre-treatment & Yes & Negative & 10.01 & Up & 5.47 & Down \\
\hline Pre-treatment & Yes & Negative & 7.82 & Up & 8.20 & Down \\
\hline Pre-treatment & Yes & Negative & 8.10 & Up & 8.56 & Down \\
\hline Pre-treatment & No & +++ & 7.79 & Up & 10.22 & Up \\
\hline Pre-treatment & No & + subset & 8.15 & Up & 9.39 & Down \\
\hline Pre-treatment & No & Negative & 7.54 & Up & 9.00 & Down \\
\hline Pre-treatment & No & +++ & 7.83 & Up & 10.16 & Up \\
\hline Post-treatment & No & + & 7.95 & Down & 10.55 & Up \\
\hline Post-treatment & No & +++ & 8.09 & Up & 9.51 & Up \\
\hline Post-treatment & No & + subset & 7.91 & Down & 7.50 & Down \\
\hline
\end{tabular}

IHC: immunohistochemistry

Note: Upregulation or downregulation of MLKL and BCL2 were determined by increased log2FC expression levels compared to median expression in controls for pre-treatment samples, and median expression in MDS patients at diagnosis for post-treatment samples. Degree of positivity was determined by intensity of expression and percentage of positive cells with a grading of + to +++ . 